\title{
A time-localized response of wave growth process under turbulent winds
}

\author{
Z. $\mathrm{Ge}^{1}$ and P. C. $\mathrm{Liu}^{2}$ \\ ${ }^{1}$ National Research Council, U.S. Environmental Protection Agency, 960 College Station Road, Athens, GA, 30605-2720, \\ USA \\ ${ }^{2}$ NOAA Great Lakes Environmental Research Laboratory, 2205 Commonwealth Blvd., Ann Arbor, MI, 48105, USA
}

Received: 31 January 2007 - Revised: 17 May 2007 - Accepted: 4 June 2007 - Published: 29 June 2007

\begin{abstract}
Very short time series (with lengths of approximately $40 \mathrm{~s}$ or $5 \sim 7$ wave periods) of wind velocity fluctuations and wave elevation were recorded simultaneously and investigated using the wavelet bispectral analysis. Rapid changes in the wave and wind spectra were detected, which were found to be intimately related to significant energy transfers through transient quadratic wind-wave and wavewave interactions. A possible pattern of energy exchange between the wind and wave fields was further deduced. In particular, the generation and variation of the strong waveinduced perturbation velocity in the wind can be explained by the strengthening and diminishing of the associated quadratic interactions, which cannot be unveiled by linear theories. On small time scales, the wave-wave quadratic interactions were as active and effective in transferring energy as the windwave interactions. The results also showed that the wind turbulence was occasionally effective in transferring energy between the wind and the wave fields, so that the background turbulence in the wind cannot be completely neglected. Although these effects are all possibly significant over short times, the time-localized growth of the wave spectrum may not considerably affect the long-term process of wave development.
\end{abstract}

Keywords. Meteorology and atmospheric dynamics (Ocean-atmosphere interactions) - Oceanography: physical (Air-sea interactions; Surface waves and tides)

\section{Introduction}

As Lavrenov (2003) puts it, the dynamic ocean is a complex system that involves five distinguishable time scales. The smallest scale, $\tau_{1}$, is the period of wave movement fluctuations, which varies from several to $10 \mathrm{~s}$. Wave movements

Correspondence to: $\mathrm{Z}$. Ge

(ge.zhongfu@epa.gov) connected with a wave group represent the second time scale, $\tau_{2}$, from 10 to $100 \mathrm{~s}$ (or $10 \sim 15 \tau_{1}$ ). The "quasi-collisions" happen on the third scale, $\tau_{3} \approx 10^{3} \mathrm{~s}$. The wave field changes occurred within a period of $3 \sim 6 \mathrm{~h}$ represent $\tau_{4} \approx 10^{4} \mathrm{~s}$. Significant changes in the wind wave spectra and in all its parameters usually occur on this time scale. The most likely largest time scale, $\tau_{5} \approx 10^{5} \mathrm{~s}$, is a synoptic range for long-term ocean wave evolution. Earlier investigations of ocean waves were primarily centered around the wave spectrum that is based on the assumption of quasi-stationarity (e.g. Hasselmann, 1962, 1963; Komen et al., 1994). This assumption essentially prohibits studies of the evolution of ocean waves on time scales smaller than $\tau_{4}$. However, Lavrenov (2001) found that the spectral maximum frequency may experience a discontinuous variation that could be explained by "quasicollisions" on $\tau_{3}$.

One may further wonder what is happening and whether it is all trivial or random over even shorter times than $\tau_{3}$. With the aid of the wavelet analysis, we investigated fieldobserved wind and wave time series as short as $40 \mathrm{~s}$, which brought our scope all the way down to the scale of $\tau_{2}$. Unlike its conventional Fourier counterpart, the wavelet-based analyses have the capacity to examine significantly shorter time series with an acceptable level of statistical noise. Over such a short time that is less than one minute or 10 typical wave periods, the governing physics might be very different from those for considerably larger time scales such as $20 \mathrm{~min}$. We envisaged that the work would provide useful information on two issues for short-time (almost instantaneous) wind-wave interactions.

One issue concerns the roles of the wave-wave and windwave resonant interactions. Phillips (1960) first studied the resonant interactions among a triad of gravity wave trains on deep water. It was found that the dispersion relation for this particular configuration does not admit any second-order resonant interaction, while the resonant interaction is possible for tertiary waves. Hasselmann was able to quantify

Published by Copernicus Publications on behalf of the European Geosciences Union. 
the energy change rate of the frequency components in a continuous wave spectrum (Hasselmann, 1962, 1963). He also found that, owing to the weak nonlinearity in the fundamental equations, the wave spectrum only undergoes a slow change with a typical interaction time of a few hours (Hasselmann, 1962). As Phillips (1974) summarized, limited by the weak nonlinearity in small-amplitude surface waves, the resonant wave-wave interactions are not only selective but also very slow. Up to date, ocean wave modeling still relies on such "slow motions" for the component of wave-wave interactions (Komen et al., 1994). Apparently, no significant energy change will take place on a small time scale such as $\tau_{2}$. In wind-generated waves, one extra mechanism would naturally be about wind forcing. With turbulent winds, the coupled wind-wave interaction is not weakly nonlinear as for pure gravity waves. In this situation, the influence of the turbulent boundary layer on the surface gravity waves is of first-order, which might admit more rapid local variation of the wave spectrum. This suspicion was never well examined with field data, due to the difficulty in processing short data records in the conventional framework of the Fourier analysis. In the present work, it became simpler using wavelet analysis.

The other major issue when time-localized variations are of interest is the role of the wind velocity fluctuations, defined here as everything in the wind velocity but the mean, which was often underestimated by existing mathematical and numerical modeling efforts in the coupled wind-wave field. For example, among the pioneering works on the numerical simulation of the air-wave interactions, Janssen $(1982,1991)$ developed a quasi-linear theory that still has great impact on the latest numerical models. Janssen's modeling neglects the effect of turbulent fluctuations, as well as nonlinearity. Admittedly, the negligence of the background turbulence in the atmospheric boundary layer did not seem to arouse serious discrepancies between the simulated and the observed results. Besides the modeling purposes, however, we still are interested in whether these assumptions or omissions reveal the true physics of the problem. The physical bases cannot be examined on a large time scale, because many meaningful events would be averaged out.

It was envisioned that this work would serve as a demonstration of the application of wavelet higher-order spectral analysis in studying ocean wave problems, and as a better understanding of the physics of wind-wave interactions on short time scales.

\section{Field observation}

\subsection{Field measurements}

The wind and wave data used in this study were recorded from the NOAA National Data Buoy Center (NDBC) 3$\mathrm{m}$ discus buoy 45011 deployed during autumn of 1997 in nearshore eastern Lake Michigan at $10-\mathrm{m}$ water depth at $43.02^{\circ} \mathrm{N}, 86.27^{\circ} \mathrm{W}$, about $1.5 \mathrm{~km}$ southwest of Grand Haven, Michigan. The NDBC 45011 buoy, similar to the ones used in the Surface Wave Dynamics Experiment (SWADE) (Weller et al., 1991), was equipped with a Datawell HIPPY 40 heave-acceleration, a pitch and roll sensor, a two-axis magnetometer, compasses, barometers, and water temperature sensors. A twin-propeller wind anemometer was mounted on the mast of the buoy $5 \mathrm{~m}$ above the design waterline of the buoy hull. Special for 45011 measurements was the simultaneous time series recording for both wind and waves at the same sampling frequency of $1.70667 \mathrm{~Hz}$. A portion of the available wind and wave time series data during a generally growing wave episode is what we have used in the following analysis.

As well known, special attention should be paid to the quality of the buoy-measured wind signals due to the fact that the motions of the buoy itself, resulting from the motion of the waves, may seriously contaminate the wind measurements. Extensive examinations have been conducted to compare the NDBC buoy winds with those recorded from other nearby buoys of the same kind, from stationary platforms (e.g. Gilhousen, 1987), and from ships (Gilhousen, 2006). Their comparisons show clearly that the 3-m buoy winds are in excellent agreement with platform winds, which are assumed to be correct, in both aspects of long-term statistics and detailed time series patterns. Of particular interest to the present study is the high correlation of 0.971 between the wind data measured from a 3-m discus buoy and from a platform (Gilhousen, 1987), implying good data quality up to sufficiently small scales. Furthermore, the wavelet-based bispectral analysis, as used here, is capable of detecting nonlinear relations even if there is some level of errors in the measured data (Van Milligen et al., 1995).

A preliminary analysis of the full-length wind and wave data sets yielded the following statistics. The average wind speed at the measuring elevation, $U_{5}$, grew from approximately $5 \mathrm{~m} \mathrm{~s}^{-1}$ to $11 \mathrm{~m} \mathrm{~s}^{-1}$ (Fig. 1). The turbulence intensity of the streamwise wind component, defined as $\sqrt{\overline{u^{2}}} / U_{5}$ ( $u$ denoting the streamwise wind velocity fluctuation) was $34.6 \%$ for $U_{5}$ being a median value of $8.5 \mathrm{~m} \mathrm{~s}^{-1}$, indicating a high level of fluctuation in the wind. Since the fluctuations were obtained by simply subtracting the average from the wind velocity time series, the wave-induced perturbation velocity should also contribute to this turbulence intensity.

\subsection{Long-term wind and wave spectra}

For an overall assessment of the long-term wave growth process, three representative episodes, starting at $t=5859 \mathrm{~s}$, $11719 \mathrm{~s}$, and $17578 \mathrm{~s}$, were taken with $2^{11}$ data points in each. Given a sampling frequency $F_{s}=1.7 \mathrm{~Hz}$, the length of each episode was $20 \mathrm{~min}$. Episode centres are indicated by arrows in Fig. 1 against a panorama of the wave growth process. Also notable from Fig. 1 is the coincident growth 

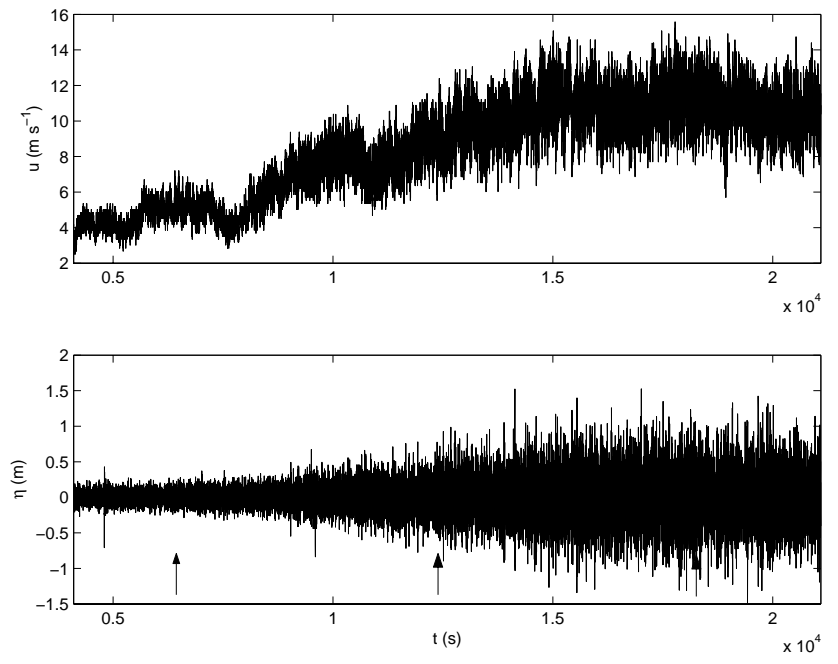

Fig. 1. Simultaneous long-term wind and wave growth processes; arrows indicate the centres of the three episodes for Figs. 2 and 3.

of both the magnitude and fluctuation of the winds. Figure 2 shows the wave spectra for the three episodes, where $\eta$ denotes the wave elevation and $P_{\eta \eta}$ denotes the (Fourierbased) wave spectrum. The three spectra curves clearly represent three sequential stages of wave growth. In particular, the equilibrium range with a -4-power law (Phillips, 1985) gradually came into form in the high frequency band from $f=0.2 \mathrm{~Hz}$ to $f=0.6 \mathrm{~Hz}$.

The Fourier spectra of the wind velocity fluctuations, $P_{u u}$, are shown in Fig. 3. One unusual characteristic that can be readily observed is the sharp spectral peak at approximately $f=0.4 \mathrm{~Hz}$ for all three episodes of wave growth. This large and sharp spectral peak seems to be superimposed on Kolmogoroff's $-5 / 3$-power region of $f>0.06 \mathrm{~Hz}$. It is known that, in the absence of strong wave impact, the atmospheric boundary layer is nearly isotropic, and the spectrum of the wind velocity fluctuations has a $-5 / 3$-power dependency in the inertial subrange (Elliott, 1972). For the present case, therefore, the large peak around $f=0.4 \mathrm{~Hz}$ should be attributed to the waves through a mechanism that is effective but has not yet been sufficiently understood.

Consequently, the wind and wave spectra have revealed that the waves, whose peak frequency is approximately $0.2 \mathrm{~Hz}$, induced a very significant frequency component in the wind fluctuations at its double frequency, $0.4 \mathrm{~Hz}$. Such highly active wave-induced frequency components in the wind at a doubled frequency of the wave peak frequency were rarely reported or studied previously. (There were doubts about the validity of the large peak at $f=0.4 \mathrm{~Hz}$ in the wind spectrum, since such a phenomenon was not often reported in the literature. The significance of this peak, i.e. it is natural but not an artifact, can be partially justified by Fig. 11. This peak is very active and effective in nonlinear interactions during stage I, while it becomes completely la-

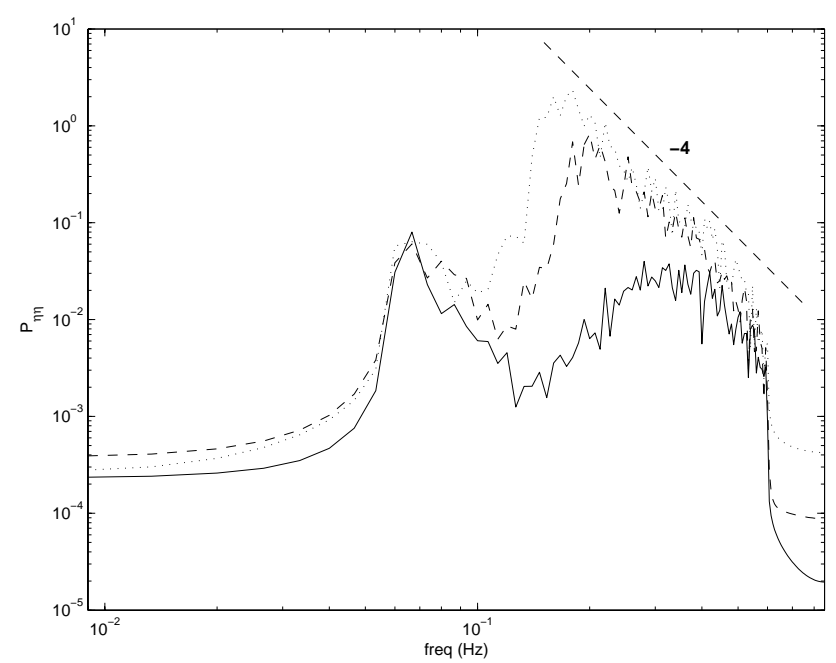

Fig. 2. Fourier wave spectra in three episodes (starting at $t=5859 \mathrm{~s}$, $11719 \mathrm{~s}$, and $17578 \mathrm{~s}$, respectively) of wave development; the duration of each episode was approximately $20 \mathrm{~min}$.

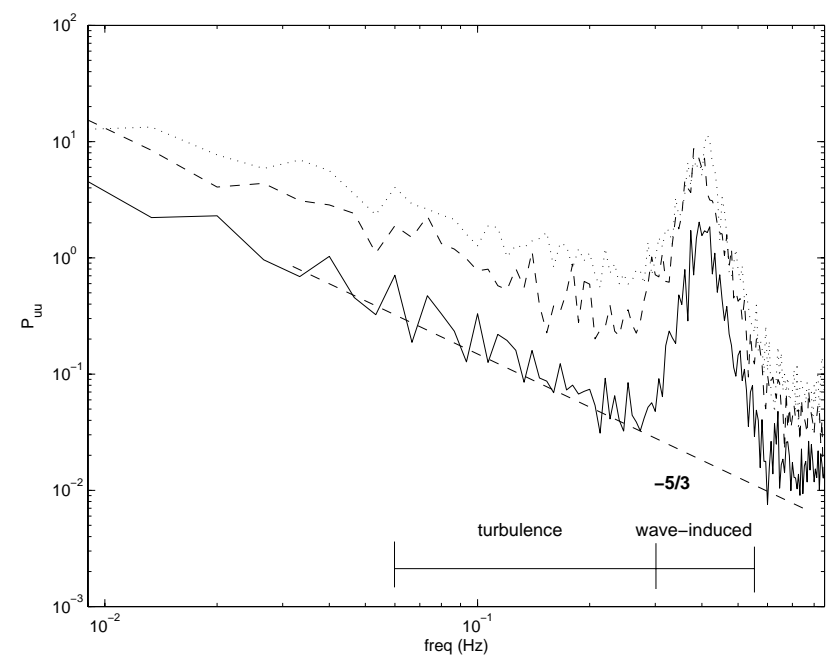

Fig. 3. Fourier wind velocity spectra in the same three episodes as those in Fig. 2.

tent during stage II. It is hence reasonable to believe that, if the peak had been created by artificial factors, such as buoy vibrations, its effect on the data would have been persistent throughout the whole data set. This is obviously contrary to the results in Fig. 11.)

Although the wind fluctuation consists of the waveinduced and the background turbulence components, they can be easily distinguished based on the wind velocity spectrum. More specifically, the narrow band around $f=0.4 \mathrm{~Hz}$ is considered to be wave-induced, while the range from $f=0.06 \mathrm{~Hz}$ to $f=0.3 \mathrm{~Hz}$ is primarily affected by the background turbulence. The two subranges are indicated in Fig. 3. 


\section{Wavelet-based spectral analysis}

In the cases where instantaneous behaviours of water waves are of particular interest, the Fourier-based analyses will result in either high variance or excessive bias in the estimated spectral moments. As a remedy, the wavelet analysis can be employed. General theories of the wavelet analysis can be found in Mallat (1998). In the remainder of this section, we only give a brief introduction to the wavelet spectral analysis that is relevant to the present work.

\subsection{Definitions}

Continuous wavelet transform was implemented. The Morlet wavelet that can be generated from the mother wavelet,

$\psi(t)=\pi^{-1 / 4} e^{i \omega_{0} t} e^{-t^{2} / 2}$

( $\omega_{0}$ set at 6.0 for the admissibility condition), through continuous translations in time and dilations of the scale was used. Using the Morlet wavelets as a basis, a real-time signal, $x(t)$, can be mapped into a two-dimensional time-scale domain through the transform

$W_{x}(a, \tau)=\int_{-\infty}^{\infty} \hat{x}(f) \hat{\psi}_{a, \tau}^{*}(f) d f$

where $W_{x}$ is the wavelet coefficient of the signal $x(t), f$ is the frequency variable that is approximately related to the scale parameter, $a$, through the conversion $f=f_{c} / a\left(f_{c}=0.9394\right.$ for the Morlet wavelets), the "hat" over a variable denotes the Fourier image of a time signal, and * denotes the complex conjugate. The temporal variations are thus preserved, which is an advantage over the conventional Fourier transform.

The wavelet power spectrum of a time series $x(t)$ over a period $T$ is defined as

$Q_{x x}(f)=\frac{1}{T f_{c} C_{g}} \int_{T} W_{x}^{*}(a, \tau) W_{x}(a, \tau) d \tau$,

where $C_{g}$ is the admissibility constant (Addison, 2002). The wavelet power spectrum is conceptually equivalent to the Fourier power spectrum based on Parseval's theorem. The two types of power spectrum are not identical to one another, in that the wavelet power spectrum is additionally determined by the chosen wavelet functions (Addison, 2002). The wavelet cross spectrum $Q_{x y}(f)$ between two time signals, $x(t)$ and $y(t)$, can be defined similarly. The wavelet linear coherence, the normalized cross spectrum to have values bounded by zero and one, is defined as

$L_{x y}(f)=\frac{\left|Q_{x y}(f)\right|^{2}}{Q_{x x}(f) Q_{y y}(f)}$.
The wavelet auto-bispectrum of a time signal, $x(t)$, has the form of

$B_{x x x}\left(f_{1}, f_{2}\right)=\int_{T} W_{x}^{*}(f, \tau) W_{x}\left(f_{1}, \tau\right) W_{x}\left(f_{2}, \tau\right) d \tau$,

with $f=f_{1}+f_{2}$. The wavelet cross-bispectrum $B_{y x x}$ between two time series, $x(t)$ and $y(t)$, can be defined similarly. They also can be normalized to have values bounded by zero and unity, referred to as the auto-bicoherence, $b_{x x x}^{2}$, and the cross-bicoherence, $b_{y x x}^{2}$, respectively. For brevity, only the definition of the wavelet cross-bicoherence is given here:

$$
\begin{aligned}
& b_{y x x}^{2}\left(f_{1}, f_{2}\right)= \\
& \frac{\left|B_{y x x}\left(f_{1}, f_{2}\right)\right|^{2}}{\left(\int_{T}\left|W_{x}\left(f_{1}, \tau\right) W_{x}\left(f_{2}, \tau\right)\right|^{2} d \tau\right)\left(\int_{T}\left|W_{y}(f, \tau)\right|^{2} d \tau\right)} .
\end{aligned}
$$

Other definitions and explanations can be found in $\mathrm{Ge}$ (2004).

For the interpretation of the results in Figs. 6-9, the crossbicoherence value, for example, at the point $\left(f_{i}, f_{j}\right)$ indicates the level of quadratic (phase) coupling among the frequency components at $f_{i}$ and $f_{j}$, respectively, in one time signal and the frequency component at $f=f_{1}+f_{2}$ in the other time signal.

\subsection{Phase coupling}

The physical meaning of the bispectral moments, both Fourier-based and wavelet-based, is intimately related to the concept of phase coupling. A phase coupling occurs, for example, when a triad of complex frequency components at $f_{1}$ and $f_{2}$, respectively, of $x(t)$ and at $f=f_{1}+f_{2}$ of $y(t)$ have a persistent relation of

$\alpha-\left(\alpha_{1}+\alpha_{2}\right)=$ constant

for a duration $T$. In this relation, $\alpha, \alpha_{1}$, and $\alpha_{2}$ are the phase angles of the spectral components at frequencies $f, f_{1}$, and $f_{2}$, respectively. During the phase coupling, the time integral in Eq. (6) will lead to a near-one bicoherence. In the remainder of this paper, the phase coupling will be referred to as quadratic coupling or quadratic interaction, in order to stress its similarity to Phillips' second-order resonant interaction among three frequency components. Despite the resemblance, phase coupling is not equivalent to the widely discussed resonant interaction, which also requires a resonant condition for the wave number vectors of the participating wave trains or frequency components. In this sense, the resonant interaction is a much more stringent type of interaction. Over a very short time, on the other hand, if the waves do not change the propagation direction significantly, the two types of interactions will be nearly identical. 


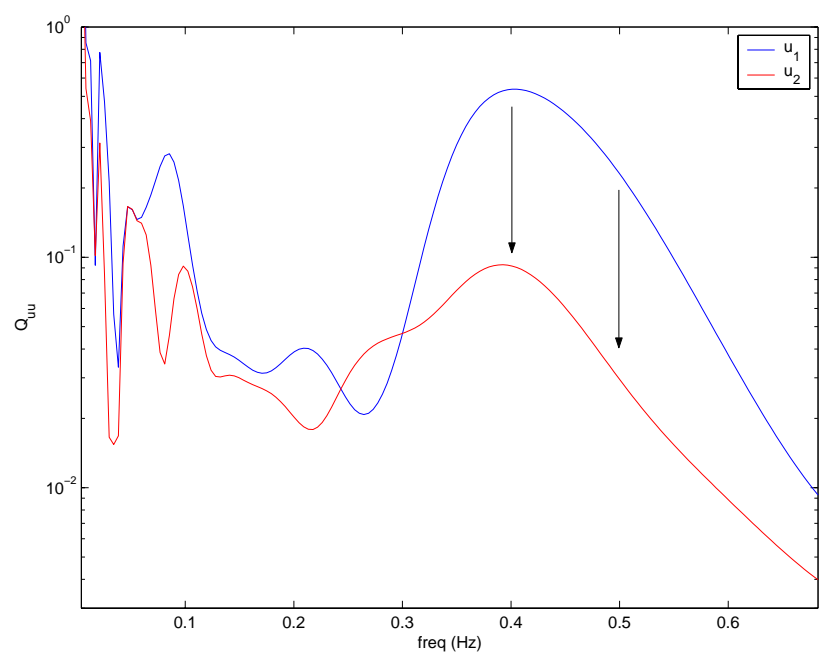

Fig. 4. Wavelet power spectra of the wind fluctuations in stages I and II; arrows highlight the changes of the energy level in major frequency components.

Unlike the bicoherence, which purely reveals the nonlinearity in the system, the bispectrum value is sensitive to the magnitudes (or modules) of the involved frequency components (see, e.g. Eq. 5). Therefore, even if the nonlinearity is not significant, the bispectrum still can have a great value when the modules of the contributing frequency components are large. The bispectrum is hence especially useful when the energy transfer among the participating components is of interest. A large bispectrum value often reveals effective energy transfer through the quadratic interaction, although the interaction itself might be weak. Conversely, a significant quadratic coupling may not facilitate effective energy transfer, simply because none of the involved components contains much energy.

\subsection{Statistical noise}

As a result of the non-orthogonality of the Morlet wavelet family, the noise level for the wavelet linear coherence is estimated as

$\varepsilon_{L}(f) \approx 2\left(\frac{F_{s}}{N f}\right)^{1 / 2}$

and that for the wavelet auto- or cross-bicoherence is estimated as

$\varepsilon_{b}\left(f_{1}, f_{2}\right) \approx\left(\frac{F_{s}}{2 N \min \left(\left|f_{1}\right|,\left|f_{2}\right|,\left|f_{1}+f_{2}\right|\right)}\right)^{1 / 2}$,

where $N$ is the number of data points in the studied time series (Van Milligen et al., 1995). It is obvious that both types of noise levels might be dominant at very low frequencies, especially when the studied time series is quite short. In such cases, inferences can be drawn only at higher frequencies, and caution must be taken when interpreting the

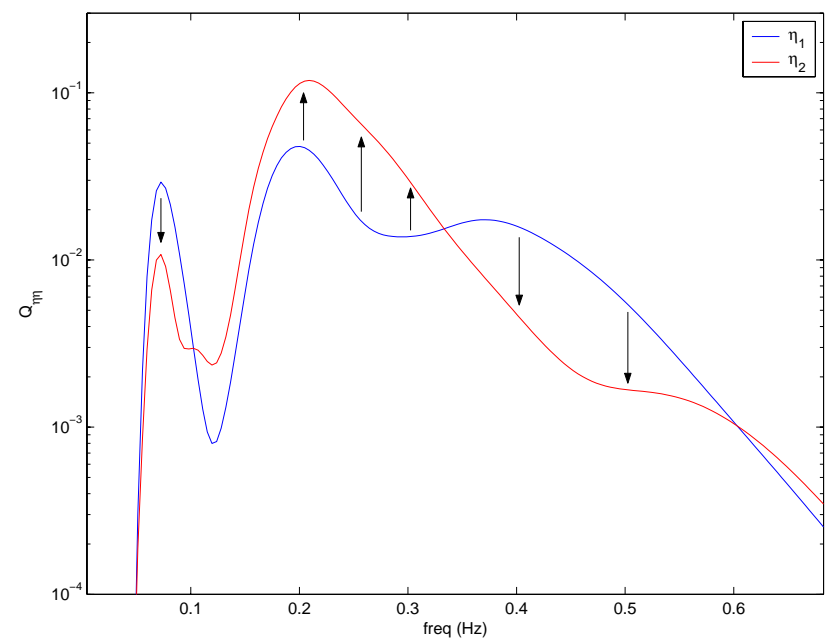

Fig. 5. Wavelet power spectra of the wave elevation in stages I and II; arrows highlight the changes of the energy level in major frequency components.

results at low frequencies. In Sects. 4 and 5, only the results of auto- and cross-bicoherence above their local noise levels are shown (Figs. 6-9). For the bispectra, we focus on intermediate and high frequency ranges where the noise level is low. The statistical noise level is explicitly shown in Fig. 10 for an illustration of its distribution.

\section{Results}

Two consecutive time series of wind velocity fluctuations, starting at $t=11593 \mathrm{~s}$, were taken from the long record. Each time series consists of only 70 data points, or approximately $40 \mathrm{~s}$ in length. They are denoted as $u_{1}$ and $u_{2}$, respectively. The two 70-point segments are referred to as stage I and stage II, respectively, hereafter. Simultaneous wave elevation series, denoted as $\eta_{1}$ and $\eta_{2}$, were also investigated.

4.1 Wavelet power spectra of the wind fluctuations and the waves

The wavelet power spectra of the wind velocity fluctuations, $Q_{u u}$, are presented in Fig. 4. In addition to the insignificant difference between the two spectra at low frequencies $(f<0.2 \mathrm{~Hz})$, the energy centered around $f=0.4 \mathrm{~Hz}$ considerably decreased by a factor of 6 from stage I to stage II, which does not appear to be due to randomness. The wavelet power spectra of the wave elevation, $Q_{\eta \eta}$, were estimated for the same two stages and are shown in Fig. 5. During the two stages, the wave energy drops at around $f=0.1 \mathrm{~Hz}$, $0.4 \mathrm{~Hz}$, and $0.5 \mathrm{~Hz}$, and increases at approximately $f=0.2 \mathrm{~Hz}$ (by a factor greater than 2). All these variations represent a rapid growth (including decay) of the wind and wave spectra, which cannot be obtained using Fourier-based analyses 

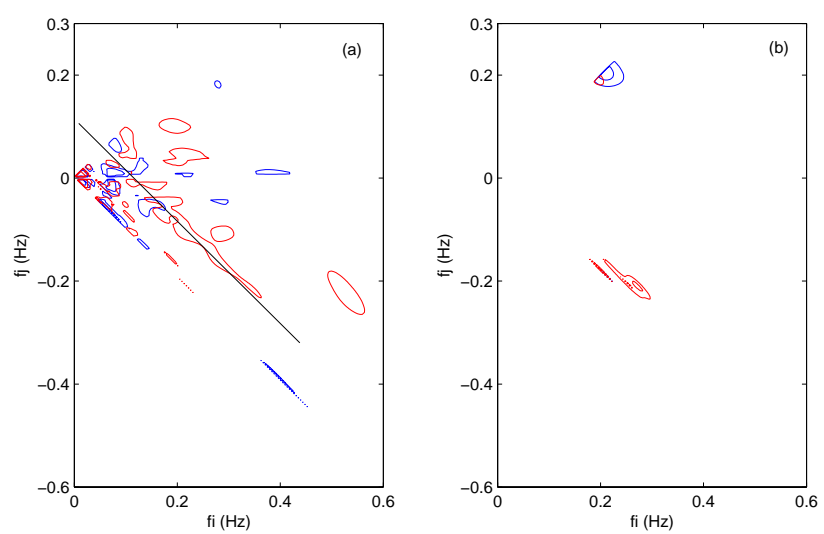

Fig. 6. Contours of (a) the wavelet bicoherence $b_{\eta \eta u}^{2}$ (contour levels: 0.45 and 0.8 ) and (b) the wavelet bispectrum $B_{\eta \eta u}$ (contour levels: 0.7 and 0.9 times of the maximum value in stage $I$ with extreme values at low frequencies eliminated) in stage I (blue contours) and stage II (red contours), respectively; all points on the black line in (a) have coordinates summed to be $0.1 \mathrm{~Hz}$.
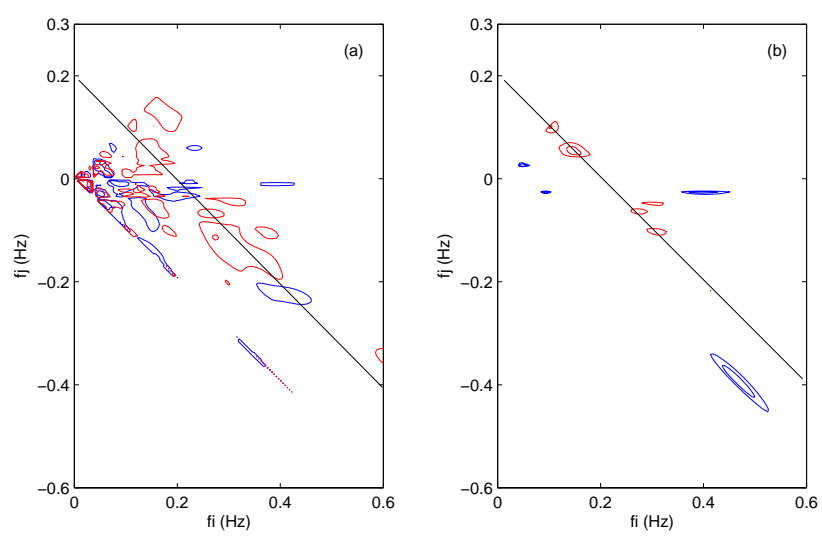

Fig. 7. Contours of (a) the wavelet bicoherence $b_{u u \eta}^{2}$ (contour levels: 0.45 and 0.8 ) and (b) the wavelet bispectrum $B_{u u \eta}$ (contour levels: 0.5 and 0.7 times of the maximum value in stage I with extreme values at low frequencies eliminated) in stage I (blue contours) and stage II (red contours), respectively; all points on the black lines in (a) and (b) have coordinates summed to be $0.2 \mathrm{~Hz}$.

over such short times. We postulate that these rapid changes in the wind and wave spectra are caused by, or at least related to, particular nonlinear interactions among frequency components.

\subsection{Nonlinear wind-wave interactions}

Figure 6 shows the wavelet bicoherence, $b_{\eta \eta u}^{2}$, and bispectrum, $B_{\eta \eta u}$, in the two stages. It is evident in panel (b) that high-level blue contours are centered around the point $(0.2$, 0.2 ), which can be interpreted as a significant energy transfer through the nonlinear (quadratic) interaction between $\eta(0.2)$ and $u(0.4)$ during stage I. (Here, for example, $\eta(0.2)$ denotes
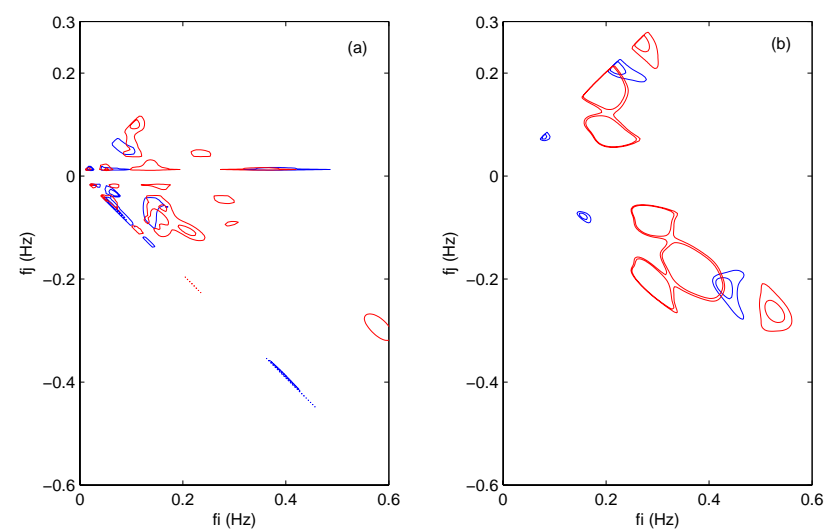

Fig. 8. Contours of (a) the wavelet bicoherence $b_{\eta \eta \eta}^{2}$ (contour levels: 0.45 and 0.8 ) and (b) the wavelet bispectrum $B_{\eta \eta \eta}$ (contour levels: 0.8 and 0.9 times of the maximum value in stage I with extreme values at low frequencies eliminated) in stage I (blue contours) and stage II (red contours).
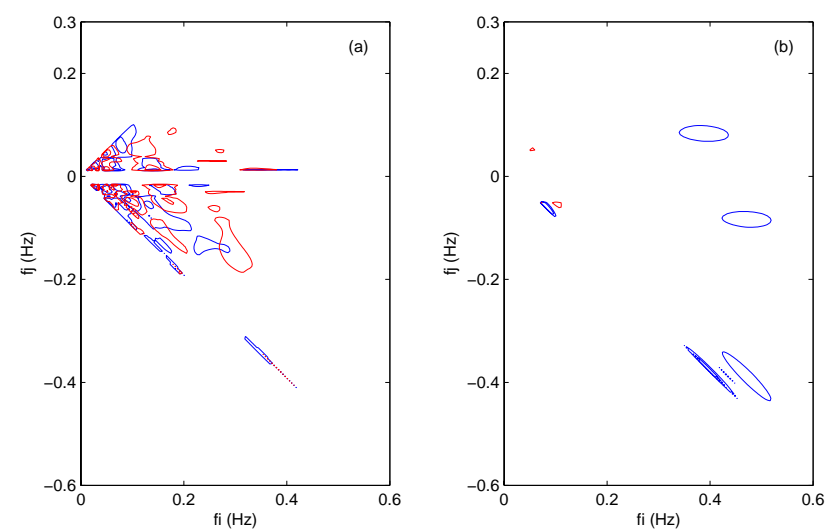

Fig. 9. Contours of (a) the wavelet bicoherence $b_{u u u}^{2}$ (contour levels: 0.45 and 0.8 ) and (b) the wavelet bispectrum $B_{u и u}$ (contour levels: 0.4 and 0.6 times of the maximum value in stage I with extreme values at low frequencies eliminated) in stage I (blue contours) and stage II (red contours).

the frequency component at $f=0.2 \mathrm{~Hz}$ in the waves, and similarly, $u(0.4)$ denotes the frequency component at $f=0.4 \mathrm{~Hz}$ in the wind fluctuations.) Since the red contours at the same point are almost absent, this interaction (energy transfer) occurred only in stage I but disappeared in stage II. The energy in $\eta(0.2)$ increased from stage I to stage II yet, meanwhile, the energy at $u(0.4)$ decreased (Figs. 4 and 5), we therefore postulate that in stage I, energy was transferred from $\eta(0.2)$ to $u(0.4)$ through the quadratic interaction at $(0.2$, 0.2 ), but, as the interaction became interrupted in stage II, $\eta(0.2)$ ceased to provide energy for $u(0.4)$, which resulted in a quick decline in the energy of $u(0.4)$. It is also observed from Fig. 6a that $u(0.1)$ became nonlinearly coupled with various frequency-component pairs in $\eta_{2}$, as indicated by the 
black line in the figure on which the sum of the coordinates is invariably 0.1 . Nevertheless, the absence of corresponding contours in Fig. $6 \mathrm{~b}$ reveals that these nonlinearities, however significant, are not effective in energy transfer. This observation agrees with Fig. 4, where the energy level at $u(0.1)$ did not change considerably.

Figure 7 shows the $b_{u u \eta}^{2}$ and $B_{\text {uu }}$ values that are complementary to the results in Fig. 6. It is noted from both $b_{u u \eta}^{2}$ and $B_{u u \eta}$ that the component, $\eta(0.2)$, became nonlinearly coupled with a broad range of frequency-component pairs in $u$ in stage II (Fig. 7a). Many of those interactions resulted in effective energy transfer, as shown in Fig. $7 b$. Since, in stage II, $\eta(0.2)$ underwent a rapid increase, it is likely that $\eta(0.2)$ received energy from these interacting frequency components, such as $u(0.05), u(0.1), u(0.15)$, and $u(0.3)$ during stage II, while these energy transfer patterns were not at work in stage I. Another evident energy transfer occurred in stage I at $(0.5,-0.4)$ in Fig. $7 b$, indicating an energy transfer supported by the nonlinear coupling among $u(0.5), u(0.4)$, and $\eta(0.1)$. This energy-transfer channel disappeared in the following stage. Noting the decreased energy in all three components from stage I to stage II, we speculate that $\eta(0.1)$ might be active in passing energy to $u(0.4)$ and $u(0.5)$ in stage I. In stage II, however, this energy-transfer channel became broken, which partially caused an energy decrease in both $u(0.4)$ and $u(0.5)$. Although the energy expense of $\eta(0.1)$ reduced in stage II, its energy level still dropped slightly due to other factors. This interpretation certainly should be consistent with all other results.

\subsection{Nonlinear interactions within the wind fluctuations and the waves}

Figure 8 shows different patterns of quadratic wave-wave interactions for the two stages. Firstly, the bispectrum value for the coupling between $\eta(0.25)$ and $\eta(0.5)$ became very large in stage II, as seen from the red contours around $(0.25$, $0.25)$ in Fig. 8b. Since the energy of $\eta(0.25)$ increased and that of $\eta(0.5)$ dropped from stage I to stage II, energy might have been transferred from $\eta(0.5)$ to $\eta(0.25)$ during stage II, but the same group of components did not transfer significant energy to each other in stage I. Secondly, the coupling around the point $(0.1,0.1)$ in Fig. $8 \mathrm{~b}$ manifests a transient energy transfer from $\eta(0.2)$ to $\eta(0.1)$ that was only effective in stage I, based on the energy changes at the corresponding frequency components across these two stages. Thirdly, the high-level contours around the point $(0.2,0.1)$ in Fig. $8 \mathrm{~b}$ reveal that the frequency component $\eta(0.1)$ started to feed significant energy into $\eta(0.2)$ and $\eta(0.3)$ in stage II through a weak quadratic interaction that cannot be seen at the corresponding point in Fig. 8a. This interpretation is in agreement with that for the coupling at $(0.5,-0.4)$ in Fig. $7 b$. It therefore appears that, in stage I, $\eta(0.1)$ provided significant energy for $u(0.4)$ and $u(0.5)$ of the turbulent wind field through the associated wind-wave interaction, and, in the subsequent stage, it continued to pass energy to $\eta(0.2)$ and $\eta(0.3)$ through a quadratic wave-wave interaction. As a result, the frequency component $\eta(0.1)$ had an energy deficit at the end of stage II. Lastly, the coupling around $(0.2,0.2)$ in Fig. $8 \mathrm{~b}$ is identified in both stages, although the strength of the energy transfer associated with this interaction appears to be greater during stage II. Based on the energy changes at $\eta(0.2)$ (increased) and at $\eta(0.4)$ (decreased) from stage I to stage II, it is very likely that $\eta(0.4)$ passed more energy to $\eta(0.2)$ through the corresponding quadratic coupling in stage II than it did in stage I.

Quadratic interactions among frequency components of the wind velocity fluctuations are shown in Fig. 9. The only quadratic coupling that allowed for effective energy transfer is indicated by the blue contours around the point $(0.4$, 0.1 ) in Fig. 9b. This quadratic coupling supported an energy transfer primarily within the wave-induced peak during stage I, since the energy in $u(0.1)$ did not seem to change much. Therefore, it is likely that $u(0.5)$ was feeding energy into $u(0.4)$ only during stage $\mathrm{I}$, and the interruption of this energy-transfer channel in stage II partially caused the decrease of the energy in $u(0.4)$. It is interesting to note that no discernible energy-containing coupling can be identified during stage II, although various nonlinear interactions were still active.

\subsection{Linear relation between the wind fluctuations and the waves}

The linear coherence $L_{u \eta}$, between the wind fluctuations and the waves in the two stages, was also estimated (Fig. 10). Similar to the quadratic interactions, the patterns of the linear coupling also vary rapidly throughout the frequency range under study. It is notable that the linear coherence declined from stage I to stage II in a broad range from $f=0.35 \mathrm{~Hz}$ to $f=0.7 \mathrm{~Hz}$, which is primarily the wave-induced range. Based on the coincidence of this range and the range in which the wind and wave energies both dropped significantly (Figs. 4 and 5), and noticing that the linear coherence is a normalized quantity that is not affected by the magnitudes of the interacting components, we believe that the linear interaction is a different mechanism for energy transfer. More specifically, the wave-induced wind velocity had a direct interplay with the waves in the same frequency range, from $f=0.35 \mathrm{~Hz}$ to $f=0.7 \mathrm{~Hz}$. This direct interaction can be represented by the level of the linear coherence, so that the wave-induced wind component would have the same frequency as the underlying waves. Furthermore, the rapid drop of the linear coherence at $f=0.2 \mathrm{~Hz}$ from stage I to stage II reveals that the linear energy transfer mechanism became absent at this frequency in stage II. Hence, the significant increase of energy in $\eta(0.2)$ during stage II was primarily a result of nonlinear interactions in that stage. 


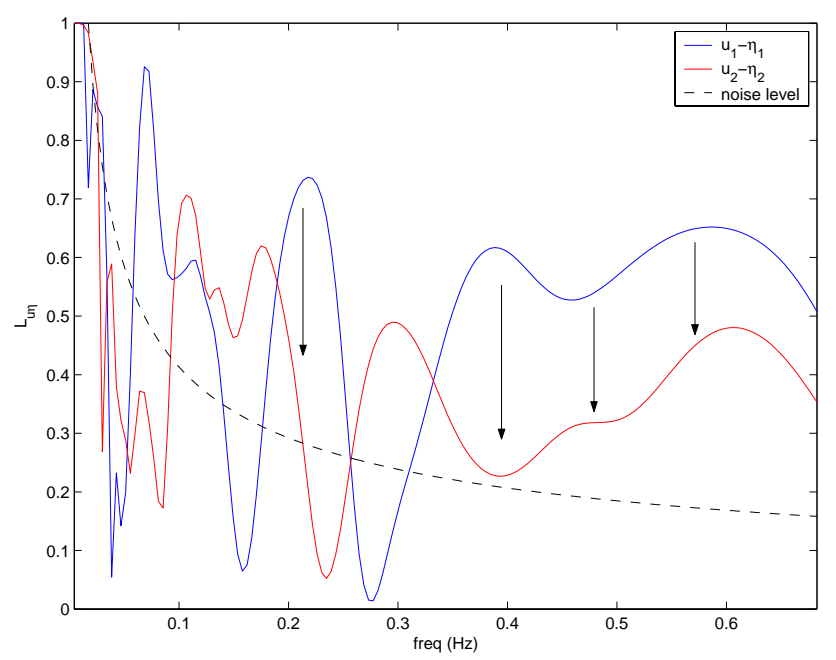

Fig. 10. Wavelet linear coherence between the wave and the wind fluctuations in stages I and II; arrows highlight the changes of the coherence level at major frequency components.

\section{Further discussion}

A possible structure of energy transfers during the two short stages was deduced in the preceding section by inspecting and comparing the wavelet-based linear coherence and bispectral moments. All detected linear and quadratic interactions among frequency components that helped transfer considerable energy are summarized in Fig. 11. During stage I, $\eta(0.1)$ and $\eta(0.2)$ were two major energy sources for the wave-induced velocity component of the wind, $u(0.4)$ and $u(0.5)$ (as revealed by Figs. 2 and 4). In contrast, during stage II, $\eta(0.2)$ ceased to energetically support the perturbation velocity in the wind, which partially resulted in the energy increase in $\eta(0.2)$. Moreover, $\eta(0.2)$ became actively coupled with various wind velocity components, $u(0.05)$, $u(0.1), u(0.15)$, and $u(0.3)$, in stage II, a dramatically different pattern of quadratic interactions. These couplings also were responsible for the energy increase of $\eta(0.2)$, although the detailed directions of energy exchange for every involved frequency component cannot be unambiguously determined. Noticing from Figs. 2 and 4 that the frequency components, $u(0.05), u(0.1), u(0.15)$, and $u(0.3)$, were mostly in the inertial subrange (the $-5 / 3$-power region) instead of the wave-induced spectral peak, these quadratic couplings represent the interplay between the wave component $\eta(0.2)$ and the background turbulence in the wind. Therefore, the wind turbulence can also nonlinearly interact with particular wave components (Fig. 7a) and effectively transfer energy (Fig. 7b). In this sense, completely neglecting the background turbulence in the wind may lead to misrepresentation of the true physics of wind-wave interactions, at least on short time scales.

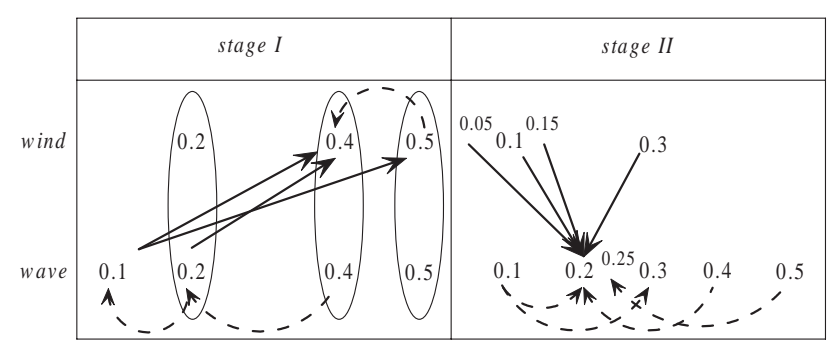

Fig. 11. Schematic of major energy transfers in stages I and II: oval link: between the wind and waves through linear coupling; solid arrow: between the wind and waves through nonlinear coupling; dashed arrow: within the wind or the waves through nonlinear coupling; unit of the frequencies: Hz.

In addition to the nonlinear wind-wave couplings, the wave-wave interaction exhibits a relay of energy transfer from the high-frequency components to the low-frequency ones, which is a common characteristic in both stages. For example, in stage I, energy was transferred from $\eta(0.4)$ to $\eta(0.2)$, and then was further passed on to $\eta(0.1)$; in stage II, similarly, energy was fed from $\eta(0.5)$ into $\eta(0.25)$, and from $\eta(0.4)$ into $\eta(0.2)$, yet accompanied by an opposite energy flux from $\eta(0.1)$ to $\eta(0.2)$ and $\eta(0.3)$. The trend of energy transfer from higher frequencies to low frequencies is consistent with the widely observed downshifting of the wave peak frequency during wave growth (Hasselmann et al., 1973). However, the process of such a transient "inverse energy cascading" in the present case appears to be realized by various types of quadratic interactions, and the process is obviously more complex than expected. It also is interesting to note, in the wave-wave interactions, that a high-frequency component tended to pass its energy to the component at its half frequency, the reason for which is not clearly understood.

In comparison to the active energy transfers through nonlinear wave-wave interactions, the quadratic coupling did not seem to be a major mechanism for energy exchange among different components in the wind velocity fluctuations. The wind field, as the anemometer could sense, can simply be viewed as isotropic turbulence superimposed by a strong wave-induced velocity component (Fig. 2). Despite the fact that the wind was full of nonlinear interactions (Fig. 9a), which probably could be attributed to the eddy motions in the turbulent boundary layer, these interactions were concentrated at low frequencies. The direct interplay of the wind turbulence and the wave-induced component was not active. This characteristic of the wind field does not disagree with the assumption made by Janssen (1989), that the waveinduced part of the wind velocity could be regarded as unaffected by the wind turbulence.

The tentative pattern of energy transfer shown in Fig. 11 also reveals a cycle of energy exchange between the wind and wave fields. For example, the general feature of stage $\mathrm{I}$ is the wave-induced wind velocity, $u(0.4)$, being supported by the 
wave component, $\eta(0.2)$. In stage II, however, energy was generally fed back from the wind turbulence to the waves, namely $\eta(0.2)$. Of course, the energy of the wind turbulence, though not fed by the wave-induced component, is typically compensated by the winds' mean flow (Tennekes and Lumley, 1972). Another part of the cycle is that the wave-induced component of the winds gained energy from $\eta(0.2)$ in stage I and then passed some back to the waves through linear coupling. This energy was in turn delivered to $\eta(0.2)$ by means of associated wave-wave interactions in stage II. Such cycles of energy transfer may only be seen locally but not significantly influence the long-term patterns of wave development, so that the current ocean wave models still appear to be accurate on larger time scales.

Besides the nonlinear interactions, the linear coupling also played an important role in exchanging energy especially within the wave-induced frequency range. This partially supports the presumed forms of the wave-induced perturbation quantities, which have the same frequency as the waves, in many previous investigations (e.g. Lee, 1972; Al-Zanaidi and Hui, 1984). In this particular case, however, the wave induction was more effectively realized through nonlinear mechanisms (Fig. 2). The absence of the wave-induced wind velocity at the wave peak frequency would make such presumed forms completely unproductive on any time scale.

We must stress again that there might be alternative energy-transfer directions for some interactions in Fig. 11. However, recording every detail of the energy transfer pattern was not our major purpose. What we have learned from the results is a network of significant energy transfer among spectral components. We have seen that, at least during short times, the quadratic wind-wave and wave-wave interactions can both play critical roles in transferring energy. On the other hand, high-value contours of the wavelet bicoherence tended not to match those of the wavelet bispectrum in the same stage, except for the red contours (i.e. in stage II) along the straight line in Figs. 7a and b. This inconsistency implies that, in most cases, the active energy transfers were not a consequence of strong nonlinear interactions, but were realized by the high energy contained in the participating components. In this sense, the findings are not essentially contrary to Phillips' resonant interaction theory, since the concept of quadratic coupling encompasses that of second-order resonant interaction (but certainly is more general), and we assume that a significant part of the detected quadratic couplings were actually resonant interactions. This consequently leads to the following thoughts: although the second-order resonance is not admissible, and the higher-order resonances are weak and slow (Phillips, 1960), their capability of passing energy among wave components in field situations might have been seriously underestimated. The superposition of various resonant interactions may bring about more rapid growth of the wave spectrum over a short time (see stage II of Fig. 11).

\section{Conclusions}

A time-localized wind-wave interaction (on Lavrenov's $\tau_{2}$ time scale) was investigated using wavelet bispectral analysis, a novel signal processing technique especially suitable for analyzing short time series. From a different perspective than many previous works, the frequency-domain analyses shed light on a few issues. It was shown that in field situations, a first-harmonic wave induced component of the turbulent winds can be strongly excited. This manifested active nonlinear interactions between the wind and the wave fields. The wavelet higher-order moments further indicated that the spectra of the wind fluctuations and waves can undergo rapid variations that are closely associated with energy exchanges between the two fields through quadratic couplings. The energy transfer pattern (Fig. 11) is characterized by two cycles of energy fluxes. In these two cycles, the wave-induced wind velocity extracted energy from the wave peak component through nonlinear couplings and then returned part of it to the waves through linear interactions. The energy was in turn passed back to the wave peak component for its continuing growth through quadratic wave-wave interactions (inverse energy cascading). In the wind fluctuations, the background turbulence, which might be continuously supported by the mean air flow through turbulence production, passed its (turbulent kinetic) energy to the wave peak components through quadratic interactions. These findings depicted fine details in the course of a long-time wave growth process (Fig. 1). From the results, we learned that all aspects, wind turbulence, nonlinearity, and the wave-wave interaction, are critical to the physics of wave growth, although some of the factors might be negligible for modeling purposes on time scales larger than $\tau_{4}$. It also is understood that the interactions identified in the present work are subject to intermittency, so that they should not bear generic statistical sense.

There certainly are other mechanisms that could contribute to the changes in the wind and wave spectra but could not be detected through the application of the waveletbased bispectral analysis. For example, the overlap of the wave-induced frequency range and the equilibrium range of the waves brings dissipation into play. The mean wind velocity, as well as the implications of wave breaking and wave grouping processes, might also affect the interaction pattern to some extent. Finally, although the field data have shown a clear picture of the nonlinear couplings, errors and uncertainties are inevitable in field measurements. To ultimately disclose such physical phenomena, carefullydesigned laboratory experiments should be conduced. These could be the goals for future work.

Disclaimer. This paper has been reviewed in accordance with the U.S. Environmental Protection Agency's peer and administrative review policies and approved for publication. Mention of trade names or commercial products does not constitute endorsement or recommendation for use. 
Acknowledgements. The authors are grateful to M. Hajj of Virginia Tech and W. Frick of EPA for their helpful discussions and support. This work also indirectly benefited from the NRC research postdoctoral associateship program.

Topical Editor S. Gulev thanks A. Babanin and another anonymous referee for their help in evaluating this paper.

\section{References}

Addison, P. S.: The illustrated wavelet transform handbook: Introductory theory and applications in science, engineering, medicine and finance, IOP Publishing Ltd., Bristol, UK, 2002.

Al-Zanaidi, M. A. and Hui, W. H.: Turbulent airflow over water waves - a numerical study, J. Fluid Mech., 148, 225-246, 1984.

Elliott, J. A.: Microscale pressure fluctuations measured within the lower atmospheric boundary layer, J. Fluid Mech., 53, 351-383, 1972.

Ge, Z.: Analysis of surface pressure and velocity fluctuations in the flow over surface-mounted prisms, Ph.D. dissertation, Virginia Polytechnic Institute and State University, Blacksburg, Virginia, USA, 2004.

Gilhousen, D. B.: A field evaluation of NDBC moored buoy winds, J. Atmos. Ocean Technol., 4, 94-104, 1987.

Gilhousen, D. B.: A complete explanation of why moored buoy winds are less than ship winds, Mariners Weather Log, 50, 1, National Oceanic and Atmospheric Administration, April 2006.

Hasselmann, K.: On the non-linear energy transfer in a gravitywave spectrum. Part 1. General theory, J. Fluid Mech., 12, 481500, 1962.

Hasselmann, K.: On the non-linear energy transfer in a gravitywave spectrum. Part 3. Evaluation of the energy flux and swellsea interaction for a Neumann spectrum, J. Fluid Mech., 15, 385398, 1963.

Hasselmann, K., Barnett, T. P., Bouws, E., Carlson, H., Cartwright, D. E., Enke, K., Ewing, J. A., Gienapp, H., Hasselmann, D. E., Kruseman, P., Meerburg, A., Muller, P., Olbers, D. J., Richter, K., Sell, W., and Walden, H.: Measurements of wind-wave growth and swell decay during the Joint North Sea Wave Project (JONSWAP), Deutches Hydrographisches Institut, Hamburg, 1973.
Janssen, P. A. E. M.: Quasilinear approximation for the spectrum of wind generated water waves, J. Fluid Mech., 117, 493-506, 1982.

Janssen, P. A. E. M.: Wave-induced stress and the drag of air flow over sea waves, J. Phys. Oceanogr., 19, 745-754, 1989.

Janssen, P. A. E. M.: Quasi-linear theory of wind-wave generation applied to wave forecasting, J. Phys. Oceanogr., 21, 1631-1642, 1991.

Komen, G. J., Cavaleri, L., Donelan, M., Hasselmann, K., Hasselmann, S., and Janssen, P. A. E. M.: Dynamics and modelling of ocean waves, Cambridge University Press, New York, NY, 1994.

Lavrenov, I. V.: Effect of wind wave parameter fluctuation on the nonlinear spectrum evolution, J. Phys. Oceanogr., 31, 861-873, 2001.

Lavrenov, I. V.: Wind-waves in oceans: Dynamics and numerical simulations, Springer-Verlag, 2003.

Lee, F. A.: Some nonlinear aspects of wind-wave interactions, J. Phys. Oceanogr., 2, 432-438, 1972.

Mallat, S.: A wavelet tour of signal processing, Academic Press, San Diego, CA, 1998.

Phillips, O. M.: On the dynamics of unsteady gravity waves of finite amplitude. Part I. The elementary interactions, J. Fluid Mech., 9, 193-217, 1960.

Phillips, O. M.: Nonlinear dispersive waves, Annu. Rev. Fluid Mech. 6, 93-110, 1974.

Phillips, O. M.: Spectral and statistical properties of the equilibrium range in wind-generated gravity waves, J. Fluid Mech., 156, 505531, 1985.

Tennekes, H. and Lumley, J. L.: A first course in turbulence, MIT press, Cambridge, MA, 1972.

Van Milligen, B. Ph., Sánchez, E., Estrada, T., Hidalgo, C., Brañas, B., Carreras, B., and García, L.: Wavelet bicoherence: A new turbulence analysis tool, Phys. Plasmas, 2, 3017-3032, 1995.

Weller, R. A., Donelan, M. A., Briscoe, M. G., and Huang, N. E.: Riding the crest: A tale of two wave experiments, B. Am. Meteorol. Soc., 72, 163-183, 1991. 